\title{
Reduction of frontal plane knee load caused by lateral trunk lean depends on step width
}

\author{
Anderson $\mathrm{J}^{\mathrm{a}}$, King $\mathrm{S}^{\mathrm{a}}$, Przybyla $\mathrm{A}^{\mathrm{b}}$, Ranganath $\mathrm{LR}^{\mathrm{c}}$ Barton $\mathrm{GJ}{ }^{\mathrm{a}}$ \\ ${ }^{a}$ Research Institute for Sport and Exercise Sciences, Liverpool John Moores University, \\ Liverpool L3 3AF, UK \\ ${ }^{\mathrm{b}}$ Department of Physical Therapy, University of North Georgia, Health \& Natural Science \\ Bldg. \#444, Dahlonega, GA 30597, USA
}

${ }^{\mathrm{C}}$ National Alkaptonuria Centre, Royal Liverpool University Hospital, Prescot Street, Liverpool

$$
\text { L7 8XP, UK }
$$

Highlights:

- Internal knee abduction moment was measured while walking with increased trunk lean

- Two strategies of increasing lateral trunk lean were observed in normal gait

- One group increased step width, the other kept normal step width with pelvic thrust

- Trunk lean over normal step width with pelvic thrust reduces the $1^{\text {st }}$ peak of moment

- Normal step width indicates better stability combined with reduced knee loading

\begin{abstract}
The internal knee abduction moment (KAM) in osteoarthritis is reduced by increased lateral trunk lean (TL). Mechanistically, this occurs as the Centre of Mass (COM) moves further over the stance leg. Since the size of the base of support constrains the COM, an associated increase in step width (SW) would be expected to maintain stability. This study tested the effects of TL on SW and KAM in healthy participants $(n=21)$ who performed normal and $6^{\circ} \mathrm{TL}$ walks. The latter was controlled via audio-visual biofeedback. We found two distinct gait strategies in TL walk: widening the step width substantially (>50\%) to permit an increase in the COM displacement (WSW, $n=13$ ), or maintaining a baseline SW and minimally displacing the COM by moving the hip/pelvic complex in the opposite direction (NSW, n=8). WSW doubled SW $(11.3 \pm 2.4$ v. $24.7 \pm 5.5 \mathrm{~cm}, \mathrm{p}<.0001)$, NSW did not change SW $(12.2 \pm 2.8 \mathrm{v}$. $13.7 \pm 4.7 \mathrm{~cm}, p>0.05)$. These two distinct gait strategies resulted in unique patterns of KAM reduction across the stance phase. NSW reduced KAM impulse significantly in the initial half $(0.08 \pm 0.02$ v. $0.06 \pm 0.02, p=.04)$ but not in the later stance phase $(0.07 \pm 0.02 \mathrm{v} .0 .07 \pm 0.04$, $p>0.05)$. WSW reduced KAM significantly in both initial $(0.11 \pm 0.03 v .0 .08 \pm 0.04, p<0.001)$ and later stance phase $(0.09 \pm 0.02 \mathrm{v}$. $0.06 \pm 0.03, p<0.001)$. KAM peak results followed the pattern of impulse. This study has revealed two distinct mechanisms for increasing lateral trunk lean which can be used to explain discrepancies in past research and in the future could be used to individualise gait re-training strategies.
\end{abstract}

\section{Key Words:}

Gait retraining; Trunk sway; Knee abduction moment; Step width; Osteoarthritis 


\section{Introduction}

Knee osteoarthritis (OA) frequently leads to pain, disability and surgical intervention. The combination of an aging population and the increase in prevalence of OA with age [1] means the disease is a growing societal burden requiring cost effective management.

The medial compartment is affected ten times more often than the lateral compartment [2] as it withstands up to $80 \%$ of the frontal plane knee load during walking [3]. During dynamic movement, the internal knee abduction moment (KAM) is accepted as a valid and reliable measure of frontal plane knee load [3,4]. The KAM calculation uses inverse dynamics [5], which mainly consists of the product of the GRF and the distance between the knee joint centre and GRF vector but also includes segmental inertial properties. A KAM is produced when the force vector passes medially to the joint centre during the stance phase [6]. The KAM peaks and impulse are increased in patients with medial compartment knee OA [7]. Further, frontal plane moments, but not sagittal plane moments, have been associated with OA disease progression over 2 years [8]. Kumar and colleagues [9] confirmed that there is a greater KAM in OA patients in comparison to healthy controls and demonstrated that the frontal plane moment is related to medial knee loading.

The KAM is recognised as a modifiable risk factor for the progression of medial knee OA [10]. Accordingly, research focuses on methods of decreasing the KAM, including lateral wedge insoles [11] and high tibial osteotomies [12]. Unfortunately, clinical trials do not show benefits from lateral wedges over prolonged periods [13] and surgery is invasive with a long recovery. Gait modification strategies have the potential to delay the progression of the disease in a non-invasive manner.

Numerous gait modifications exist, including altering the foot progression angle, increasing medial knee thrust and increasing lateral trunk lean. In a comparison of these strategies, lateral trunk lean was the most successful method of decreasing KAM in almost half the subjects [14]. Reductions of $30 \%, 38 \%$ and $28 \%$ for the KAM impulse, $1^{\text {st }}$ peak and $2^{\text {nd }}$ peak, respectively, were reported in comparison to normal walking. A review considering 14 gait modification strategies found lateral trunk lean and the use of a walking cane to most consistently decrease KAM [15]. However, some people have reservations about using a cane, based on its appearance [16]. Modifying trunk lean removes this obstacle. In the natural gait of patients with medial compartment knee OA, lateral trunk lean was shown to explain $13 \%$ of the variance in KAM when toe out angle and speed were controlled for [17]. This also suggests that gait modification strategies occur naturally in this population, adding an increased need to understand the impact these have on the affected and unaffected joints. 
Furthermore, increasing lateral trunk lean has been shown to reduce KAM more than that previously reported with high tibial osteotomies [18].

The mechanism behind the increasing TL causing a decrease in KAM hinges on the alteration in moment arm length. The increased TL moves the centre of mass (COM) further over the stance leg, causing the GRF vector to move laterally, shortening the moment arm of the knee joint [18], resulting in a decreased moment. However, the COM movement is constrained by the size of the base of support, and must remain within the borders of the step width (SW) $[19,20]$. As humans walk on a relatively narrow base of support with up to two thirds of an individual's mass carried superior to the waist [21], it is hypothesised that an increase in SW would occur alongside the increase in TL to prevent imbalance. Despite this, studies investigating TL do not generally report SW. Therefore, the aim of this study was to test the effects of increased trunk lean on consequential alterations in step width and the impact it has on the KAM. It is predicted that an increase in trunk lean will be accompanied by an increase in step width.

\section{Method}

\section{Participants}

Participants with no musculoskeletal or neurological injuries or impairments were recruited from a university population. Twenty-one subjects took part (male: 11, female: 10, $23 \pm 2$ years, $68 \pm 8 \mathrm{~kg}, 1.74 \pm 0.06 \mathrm{~m}$ ). Ethical approval was granted by the University Ethics Committee (P14SEC020).

\section{Set-up}

A nine-camera (T10/T160, Vicon Motion Analysis Inc., Oxford, UK) motion analysis system $(100 \mathrm{~Hz})$ was centred around two adjacent force plates (Kistler 9281B; Kistler Instruments Ltd. Winterhur, Switzerland, $1000 \mathrm{~Hz}$ ) and collected using Vicon Nexus (v1.85). Participants were barefoot to eliminate the impact of footwear on KAM [22]. Height, weight, knee width and ankle width were measured. A Helen-Hayes model [23] was adapted to include the trunk (markers on iliac crest, C8, Th7, xyphoid process, jugular notch, and acromion process). The HelenHayes thigh wand orientation was checked using the Vicon plug-in-gait model to ensure the frontal plane knee angle did not exceed $\pm 10^{\circ}$ as these values are anatomically unrealistic and thus likely a result of cross talk from a misalignment of the knee flexion/extension axis.

Data Collection 
Participants were tested in two conditions: walking with normal trunk lean (NTL) and $6^{\circ}$ of lateral trunk lean (LTL), similar to that previously used [24]. The NTL was tested first to gauge self-selected walking speed. Trials were accepted if walking speed lay within $10 \%$ of this value.

The TL movement was controlled using an inertial sensor (Xsens MTw, Xsens Technologies B.V.), attached on the xiphoid process. A custom biofeedback software application was created (D-Flow v3.18.1, Motekforce Link, Amsterdam). Visual feedback was presented on a screen at the end of the walkway. A blue line rotated about a fixed axis in the frontal plane as the participant walked, mirroring their trunk lean. Participants had a zone that spanned $4-8^{\circ}$ bilaterally of central that they were instructed to aim for. In this zone, the line changed to white and a beep was heard (Figure 1). If they went past the $8^{\circ}$ line, a second error noise was heard. Demonstrations and feedback were provided. The participants practiced the trunk lean walk until a consistently correct strategy was adopted. The sensor was reset to $0^{\circ}$ when the participant was stood statically. This was reset after each trial to minimise drift. . No instructions were given in regards to step width or the lower limbs. Trials were repeated if any of the steps exceeded or did not reach the required level of trunk lean and/or if the right foot did not cleanly contact the force plate. Three successful trials were collected for each condition.

[Figure 1]

Data analysis

Each trial was cropped to a single stride exported into Visual3D (version 5; C-Motion, Inc., Germantown, MD, USA) and filtered (low pass, fourth-order Butterworth filter at $8 \mathrm{~Hz}$ ). A Helen-Hayes lower limb model with an additional trunk segment was applied. Knee and ankle joint centres were defined as half of their measured width medial to the lateral marker. The trunk was defined using the acromion and iliac crest markers with the remaining used for tracking. Lateral trunk lean angle was calculated relative to the laboratory reference system, with all angles recorded from the optoelectronic system rather than the IMU. Right leg data was analysed. SW was defined as the medio-lateral distance between the heel markers during initial contact with the ground (double support) at each step. KAM impulse, calculated as the area under the curve, was exported directly from Visual3D. COM displacement was exported from Visual3D into Microsoft Excel (2013). Any small deviation from walking in a straight line caused a trend in the sideways position of the COM which was removed for each walk by subtracting the regression line that was fitted on the COM sideways position plotted against time. The average of the 3 trials were calculated for each participant.

Statistical Analysis: 
A t-test was used to analyse the effects of biofeedback on TL, gait velocity and to identify the wide step width gait strategy in response to increased lateral trunk lean on an individual basis. Other dependent variables were subjected to a 2-way mixed model ANOVA. For the analysis of step width, COM displacement, and time at which peak trunk lean was reached, a $2 \times 2$ design was used; between subject factor: gait strategy (normal/narrow step width: NSW or wide step width: WSW), within subject factor:, lateral trunk lean (normal: NTL or increased lateral: LTL). For the analysis of KAM variables a 2x4 design with the gait strategy) was used as the between-subject factor, and the lateral trunk lean at the beginning and at the latter phase of stance as the within-subject factors. All statistical tests were completed using JMP Pro 11 (SAS Institute Inc., Cary, NC, USA).

\section{Results}

The use of biofeedback to increase lateral trunk lean of healthy participants was successful. Peak trunk lean angle showed a significant increase from $2.74 \pm 2.05^{\circ}$ in the NTL to $7.99 \pm 2.53^{\circ}$ in the LTL $\left(t_{20}=7.47, p<.0001\right)$. Gait velocity was maintained between conditions (NTL= $1.59 \pm 0.10 \mathrm{~ms}^{-1}$ vs. LTL=1.61 $\pm 0.09 \mathrm{~ms}^{-1}, \mathrm{t}_{20}=.89, \mathrm{p}=.39$ ).

Two distinct gait strategies emerged when walking with $6^{\circ}$ TL: maintaining a normal step width (NSW) between conditions $(n=8, p<0.05)$ or widening step width (WSW) in response to the increasing TL $(n=13, p>0.05)$. The two-way mixed model ANOVA (between subject: gait strategy (NSW and WSW), within subject: trunk lean (NTL and LTL)) revealed a significant interaction $\left(F_{1,19}=28.83, p<.0001, \eta=.60\right)$. Post-hoc comparisons showed that in the $6^{\circ} \mathrm{TL}$, the WSW group increased step width $(11.31 \pm 2.4$ to $24.7 \pm 5.5 \mathrm{~cm}, p<.0001)$ whereas the NSW group maintained a constant step width (NTL=12.2 \pm 2.8 vs. $L T L=13.7 \pm 4.7 \mathrm{~cm}, \mathrm{p}>.05$ ). Individual alterations in step width can be seen in Figure 2.

[Figure 2]

The two modes of walking with a trunk lean had a large impact on the KAM curves, as quantified by KAM impulse in the initial and latter phases of gait. The two-way mixed model ANOVA revealed a significant interaction $\left(F_{3,17}=6.22, p<0.005, \eta=0.52\right)$, with the post-hoc demonstrating the NSW group significantly reduced KAM impulse in the initial half $(p=0.04)$, but not in the latter half of the stance phase ( $p>0.05)$. The WSW group reduced the impulse for both halves of the stance phase $(p<0.001)$. A similar pattern was seen in KAM peak amplitude, with a significant interaction $\left(F_{3,17}=8.52, p<0.001, \eta=0.60\right)$. The WSW group reduced initial and latter KAM peaks $(p<.004)$, but the NSW reduced initial KAM peak only $(p<.05)$, Table 1. 
[Table 1]

The NSW and WSW groups varied in the timing of peak trunk lean in the LTL walk. The ANOVA revealed a significant interaction between gait strategy and time to peak trunk lean $\left(F_{1,19}=11.60, p<0.003, \eta=0.38\right)$. Post-hoc comparison showed the WSW group significantly delayed the time of the peak trunk lean $(p<0.001)$, but the NSW group showed no changes $(p>0.05)$.

The centre of mass (COM) displacement showed neither an interaction $\left(F_{1,19}=0.39, p>0.05\right.$, $\left.\eta^{2}=0.02\right)$ nor a main effect of gait strategy $\left(F_{1,19}=1.09, p>0.05, \eta^{2}=0.05\right)$. The effect of trunk lean condition was significant $\left(F_{1,19}=20.82, p<0.001, \eta^{2}=0.52\right)$ with COM displacement increased from the NTL to LTL walk (Table 1). However, step width increases are continuous, with a wide range of increases seen, which may contribute to the lack of significance between groups. The mean COM displacement demonstrates the difference between groups (Figure 3). The COM displacement increases from NTL to LTL walk to a greater extent in the WSW group in comparison to the NSW.

\section{Discussion}

This study has revealed two methods by which a cohort of healthy individuals achieves an increased TL: one increased step width (WSW) whilst the other maintained the same step width (NSW). When walking with $6^{\circ} \mathrm{TL}$, the WSW group delayed the timing of peak trunk lean in comparison to their normal walk, whereas the NSW group did not. Further, there is an indication that a wider step width is associated with an increased COM displacement. These group differences impacted the KAM, with the WSW group demonstrating reductions in both the initial and later impulse and peaks, whereas the NSW only reduced the initial KAM variables.

The mechanism behind the increase in TL differs for each group. In the WSW group, increasing step width reduces the restraint on the COM displacement [20] allowing the COM to displace further over the stance leg whilst remaining within the increased base of support. The decrease in frontal plane knee moments occurs as the GRF vector shifts laterally, creating a shorter moment arm [18].

In the NSW group, a different strategy is employed in which the COM is displaced to a lesser extent (Figure 3). Due to the narrow base of support, this can only be possible by rearranging other body segments around the COM. As the trunk (two thirds of an individual's mass [21]) leans over the support side, the rest of the body must shift to the opposite side to prevent a large COM displacement and imbalance. This movement most likely occurs as a pelvic thrust 
forcing the whole leg to be tilted away from the support side (Figure 2). Such tilting of the leg is not dissimilar from the medial knee thrust described by Simic et al. [15] which moves the knee closer to the GRF vector, reducing the frontal plane moment arm and therefore the KAM. The decreased COM displacement would most likely lead to a more efficient gait as increasing step width when walking has been shown to result in increases in mechanical (54\%) and metabolic (45\%) costs [25].

The NSW group reached peak TL earlier in the gait cycle than the WSW group. As the TL in the NSW group peaks at a similar time to the $1^{\text {st }}$ peak KAM, the main effect of the TL occurs here. After the maximum TL is reached, the trunk moves back towards a neutral position. At the time of the $2^{\text {nd }}$ peak KAM (about $75 \%$ of stance phase), TL values are similar to that when walking with a normal trunk lean, explaining why KAM moment is not reduced. The timing of maximum TL has previously been shown to influence $1^{\text {st }}$ peak KAM reductions, with the optimal $1^{\text {st }}$ peak KAM occurring when peak TL coincided with the time of $1^{\text {st }}$ peak KAM [26]. Comparatively, in the WSW group, the peak TL value occurs at approximately mid stance causing a shallower increase and decrease in TL angle. This causes the COM to be displaced more laterally over the stance leg at the time of both KAM peaks in comparison to when walking normally. Therefore a reduction in KAM variables at both time points occurs.

Previous literature has failed to report SW. However, there are result discrepancies that could be explained by these two gait strategies. Hunt and colleagues [27] found significant reductions for knee frontal plane moments were only seen in the first peak KAM. Although SW was not reported, peak TL occurred at approximately $25 \%$ of the stance phase, following the same pattern as the data produced in the NSW group. Further, the average $1^{\text {st }}$ peak decrease of $25 \%$ was similar to the $21 \%$ average decrease reported for the NSW group. Caldwell and colleagues [28] similarly reported a $32 \%$ decrease in $1^{\text {st }}$ peak KAM and a $7 \%$ decrease in $2^{\text {nd }}$ peak KAM (3\% decrease in this study).. Conversely, some studies found reductions across all three variables, such as that by Gerbrands and colleagues [14], who reported decreases of between $28-38 \%$ for all three KAM variables. The $2^{\text {nd }}$ peak decrease of $38 \%$ is comparable to the $29 \%$ reduction in the WSW group in this study.

It is accepted in the literature that modification to any lower limb joint during single-limb standing will impact other joints in the kinetic chain [29]. Adopting a gait strategy that spreads the excessive load from the affected joints over a number of different joints could reduce the likelihood of any joints crossing a threshold that would cause further problems to arise, such as increased local forces, leading potentially to back pain [30] or increased energy cost [31]. The use of the narrow step width combined with the trunk lean and necessary pelvic thrust 
may well act as such a distribution mechanism across multiple joints, although this remains to be tested.

It must be noted that this study has some limitations. As the two groups were not expected apriory, it was impossible to control the number of, or any factors between, participants in each group. Our simplified 3D body model included neither the arms nor the head, thus limiting the COM calculation accuracy. Further investigation needs to confirm the link between gait strategy and COM displacement as well as to quantify the extent to which the pelvic and hip kinematics change, to verify the suggested movement pattern reported here. The use of young, healthy participants in this study enabled identification of a new gait strategy and we believe our results provide evidence for developing and testing this paradigm in clinical populations, i.e. with knee OA.

The results of this study have various applications. They provide a plausible explanation for previous inconsistencies in the literature. We have identified that step width must be considered and controlled for in future research. This research hints at the future ability to select gait modification strategies based on which phase of the gait cycle requires modification, thus individualising gait retraining strategies. For example, in patients with severe osteoarthritis typically the first peak of the KAM is increased [32] and so they could benefit from the NSW trunk lean with an opposite pelvic thrust while maintaining stability and energy expenditure due to unchanged sideways movement of the COM.

\section{Conclusions}

This study has identified two mechanisms for increasing trunk lean in healthy participants: increasing step width to enable an increase in COM displacement or by maintaining step width and using a pelvic thrust in the opposite direction to the trunk lean to maintain normal COM displacement. The delay in peak trunk lean in the WSW group allows a decrease in KAM throughout the stance phase in comparison to the NSW group in which the reduction is focused on the initial half potentially making it a beneficial gait modification in severe OA. An added advantage of NSW is that it provides more stability as walking with trunk sway is possible over a normal step width, and the energy cost of walking is not expected to increase. 


\section{References:}

[1] Corti, M.C., C. Rigon, Epidemiology off osteoarthritis: prevalence, risk factors and functional impact. Aging Clin. Exp. Res. 15 (2003) 259-363.

[2] Ahlback, S., Osteoarthritis of the knee. Acta Radiol. 277 (1968) 70-72.

[3] Dayal, N., A. Chang, D. Dunlop, K. Hayes, R. Chang, J. Song, L. Torres, L. Sharma, The natural history of anteroposterior laxity and its role in knee osteoarthritis progression. Arthritis Rheum. 52 (2005) 2343-49.

[4] Birmingham, T.B., M.A. Hunt, I.C. Jones, T.R. Jenkyn, J.R. Griffin, Test-retest reliability of the peak knee adduction moment during walking in patients with medial compartment knee osteoarthritis. Arthritis Rheum. 25 (2007) 789-97.

[5] Richards, R., J.C., van den Noort, J. Dekker, J. Harlaar, Gait Retraining with real-time Biofeedback to reduce Knee adduction moment: systematic review of effects and methods used. Arch. Phys. Med. Rehabil. [in press].

[6] Vincent, K.R., B.P. Conrad, B.J. Fregly, H.K. Vincent, The pathophysiology of osteoarthritis: a mechanical perspective on the knee joint. PM \& R. 4 (2012) S3-S9.

[7] Thorp, L.E., D.R. Sumner, J.A. Block, K.C. Moisio, S., Shott, M.A. Wimmer. Joint loading differs in individuals with mild compared with moderate medial knee osteoarthritis. Arthritis Rheum. 54 (2006) 3842-49.

[8] Chang, A.H., K.C. Moisio, J.S. Chmiel, F. Eckstein, A. Guermazi, P.V. Prasad, Y. Zhang, O, Almagor, L. Belisle, K.Hayes, L. Sharma. External knee adduction and flexion moments during gait and medial tibiofemoral disease progression in knee osteoarthritis. Osteoarthr. Cartil. 23 (2015) 1099-106.

[9] Kumar, D., K.T. Manal, K.S. Rudolph. Knee joint loading during gait in healthy controls and individuals with knee osteoarthritis. Osteoarthr. Cartil. 21 (2013) 298-305.

[10] Miyazaki, T., M. Wada, H. Kawahara, M. Sato, H. Baba, H. Shimada. Dynamic load at baseline can predict radiographic disease progression in medial compartment knee osteoarthritis. Arthritis Rheumatol. 61 (2002) 617-22. 
[11] Hinman, R.S., K.A. Bowles, B.B. Metcalf, T.V. Wrigley, K.L. Bennell, Lateral wedge insoles for medial knee osteoarthritis: effects on lower limb frontal plane biomechanics. Clin. Biomech. 27 (2012) 27-33.

[12] Prodromos, C.C., T.P. Andriacchi, J.O. Galante. A relationship between gait and clinical changes following high tibial osteotomy. J. Bone Joint Surg. 67 (1985) 1188-94.

[13] Bennell, K.L., K.A. Bowles, C. Payne, F. Cicuttini, E. Williamson, A. Forbes, F. Hanna, M. Davies-Tuck, A. Harris, R.S. Hinman. Lateral wedge insoles for medial knee osteoarthritis: 12 month randomised controlled trial. Br. Med. J. 18 (2011) d2912.

[14] Gerbrands, T.A., M.F. Pisters, B. Vanwanseele. Individual selection of gait retraining strategies is essential to optimally reduce medial knee load during gait. Clin. Biomech. 29 (2014) 828-34.

[15] Simic, M., R.S. Hinman, T.V. Wrigley, K.L. Bennell, M.A. Hunt. Gait modification strategies for altering medial knee joint load: a systematic review. Arthritis Care Res. 63 (2011) 405-26.

[16] Shrier, I., D.E. Feldman, M.C. Gaudet, M. Rossignol, D. Zukor, M. Tanzer, C. Gravel, N. Newman, R. Dumais, Conservative non-pharmacological treatment options are not frequently used in the management of hip osteoarthritis. J. Sci. Med. Sport. 9 (2006) 81-86.

[17] Hunt, M.A., T.B. Birmingham, D. Bryant, I. Jones, J.R. Griffin, T.R. Jenkyn, A.A. Vandervoort. Lateral trunk lean explains variation in dynamic knee joint loads in patients with medial compartment knee osteoarthritis. Osteoarthr. Cartil. 16 (2008) 591-99.

[18] Mündermann, A., J.L. Asay, L. Mündermann, T.P. Andriacchi, Implications of increased medio-lateral trunk sway for ambulatory mechanics. J. Biomech. 41 (2008) 165-170.

[19] MacKinnon, C.D., D.A. Winter, Control of whole body balance in the frontal plane during human walking. J. Biomech. 26 (1993) 633-44.

[20] Horak, F.B., Postural orientation and equilibrium: what do we need to know about neural control of balance to prevent falls? Age Ageing. 32 (2006) ii7-ii11.

[21] Krebs, D.E., D. Wong, D. Jesevar, P. O'Riley, A.Hodge, Trunk kinematics during locomotor activities. Phys. Ther. 72 (1992) 505-14.

[22] Radzimski, A.O., A. Mündermann, G. Sole. Effect of footwear on the external knee adduction moment - a systematic review. The Knee. 19 (2012) 163-175. 
[23] Davis, R.B., S. Ounpuu, D. Tyburski, J.R. Gage, A gait analysis data collection and reduction technique. Hum. Mov. Sci. 10 (1991) 575-87.

[24] Simic, M., M.A. Hunt, K.L. Bennell, R.S. Hinman, T.V. Wrigley. Trunk lean gait modification and knee joint load in patients with medial knee osteoarthritis: the effect of varying trunk lean angles. Arthritis Care Res. 64 (2012) 1545-53.

[25] Donelan, J.M., R. Kram, Mechanical and metabolic determinants of the preferred step width in human walking. Proceedings of the Royal Society of London B: Biological Sciences. 268 (2001) 1985-92.

[26] Mündermann, A., L. Mündermann, T.P. Andriacchi. Amplitude and Phasing of trunk motion is critical for the efficacy of gait training aimed at reducing ambulatory loads at the knee. J. Biomech. Eng. 134 (2012) 011010.

[27] Hunt, M.A., M. Simic, R.S. Hinman, K.L. Bennell, T.V. Wrigley. Feasibility of a gait retraining strategy for reducing knee joint loading: increased trunk lean guided by real-time biofeedback. J. Biomech. 44 (2011) 943-47.

[28] Caldwell, L.K., L.L. Laubach, J.A. Barrios. Effect of specific gait modifications on medial knee loading, metabolic cost and perception of task difficulty. Clin. Biomech. 28 (2013) 64954.

[29] Takacs, J., M.A. Hunt. The effect of contralateral pelvic drop and trunk lean on frontal plane knee biomechanics during single limb standing. J. Biomech 45 (2012) 2791-96.

[30] van den Noort, J.C., I. Schaffers, J. Snijders, J. Harlaar. The effectiveness of voluntary modifications of gait pattern to reduce the knee adduction moment. Hum. Mov. Sci. 32(2013) 412-24.

[31] Takacs, J., A.A. Kirkham, F. Perry, J. Brown, E. Marriott, D. Monkman, J.Havey, S. Hung, K.L. Campbell, M.A. Hunt. Lateral trunk lean gait modifications increases the energy cost of treadmill walking in those with knee osteoarthritis. Osteoarthr. Cartil.22 (2014) 203-209.

[32] Mündermann, A., C.O. Dyrby, T.P. Andriacchi, Secondary gait changes in patients with medial compartment knee osteoarthritis: increased load at the ankle, knee, and hip during walking. Arthritis Rheum. 52 (2005) 2835-44. 
Figure 1: Biofeedback system. Image A represents the screen image when the participant was stood straight. Image B shows the participant reaching the required zone to the left. The blue line has turned white and the end of the line is within the green circles zone. A noise would be heard at either green circle. Trials were only accepted if the line reached, but did not go over the correct zone.

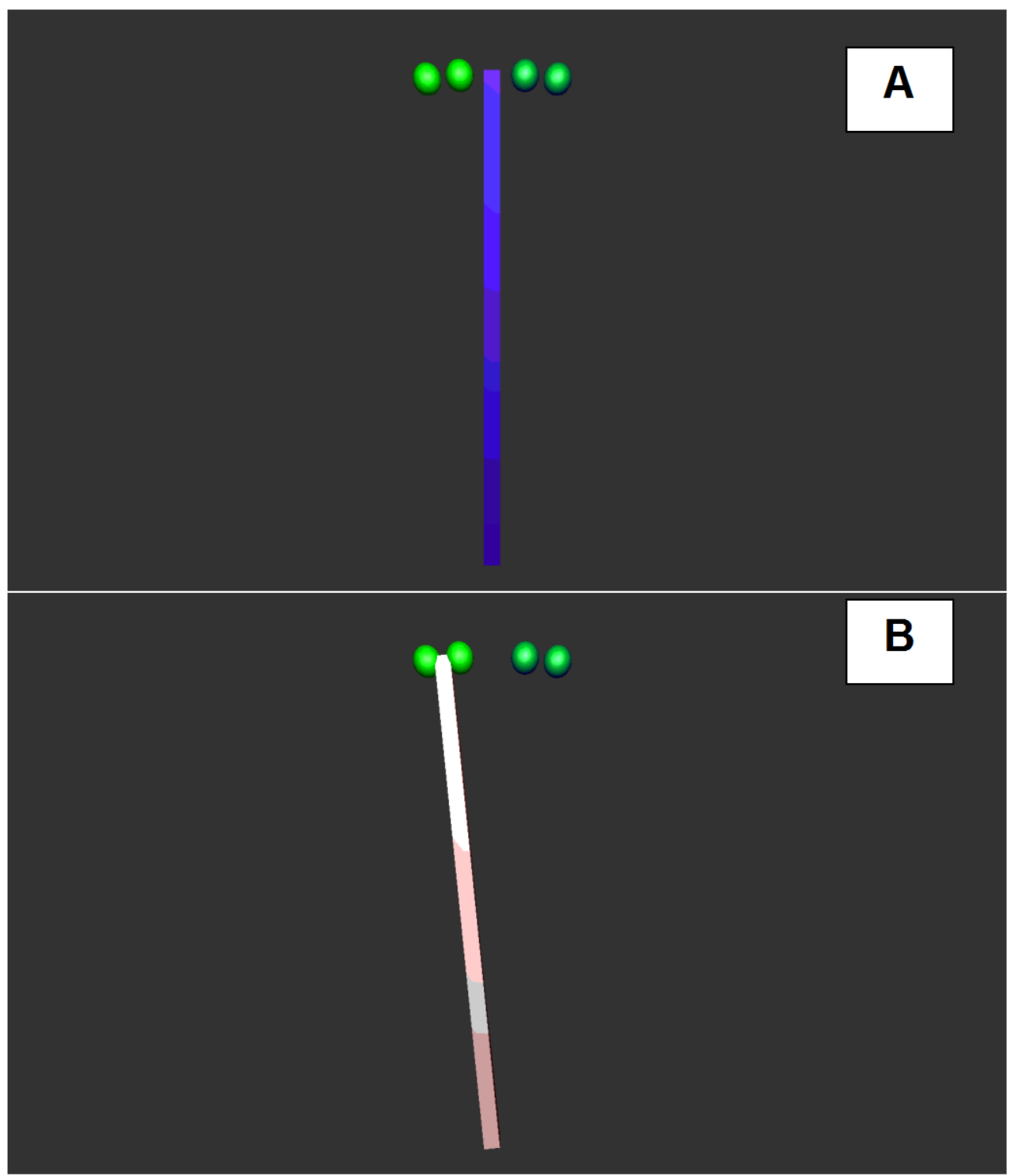


Figure 2: Diagrams of the two gait strategies adopted when lateral trunk lean angle is increased, with corresponding lateral trunk lean angle and KAM curves. In the charts solid lines display means and shaded areas represent \pm standard deviation.
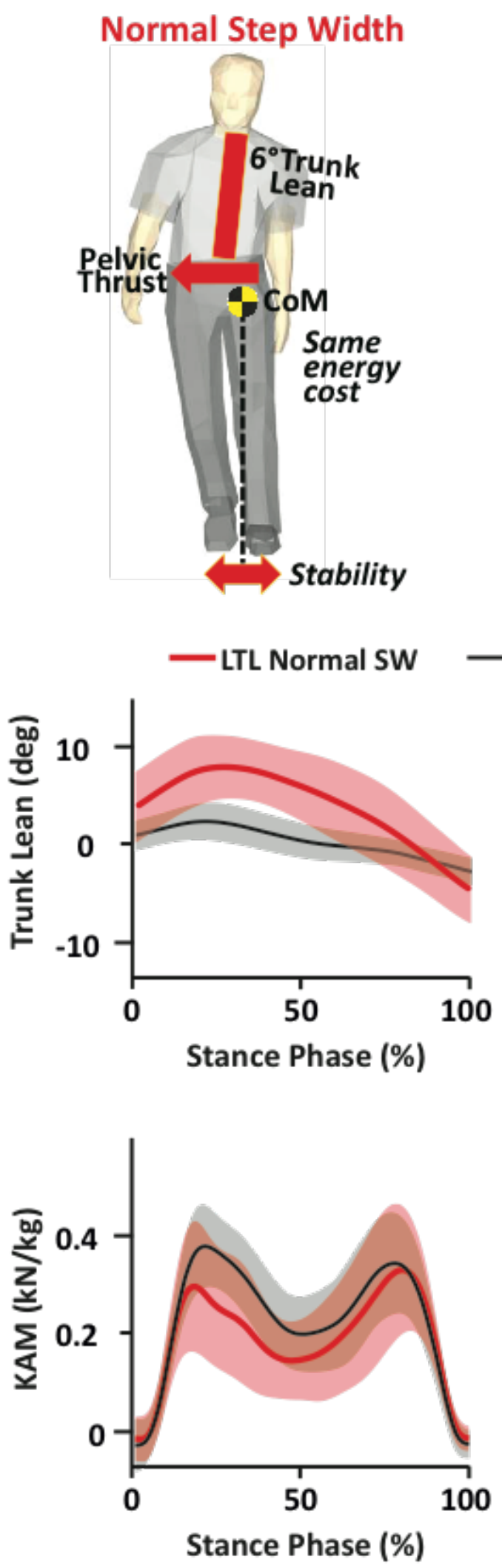

\section{Wide Step Width}

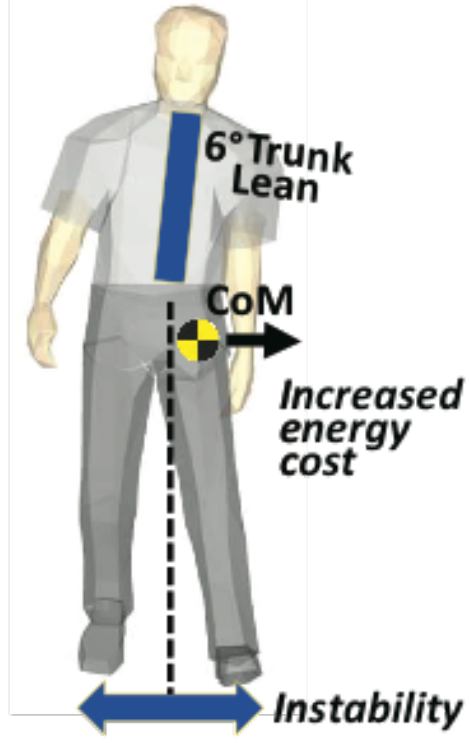

NTL

— LTL Wide SW
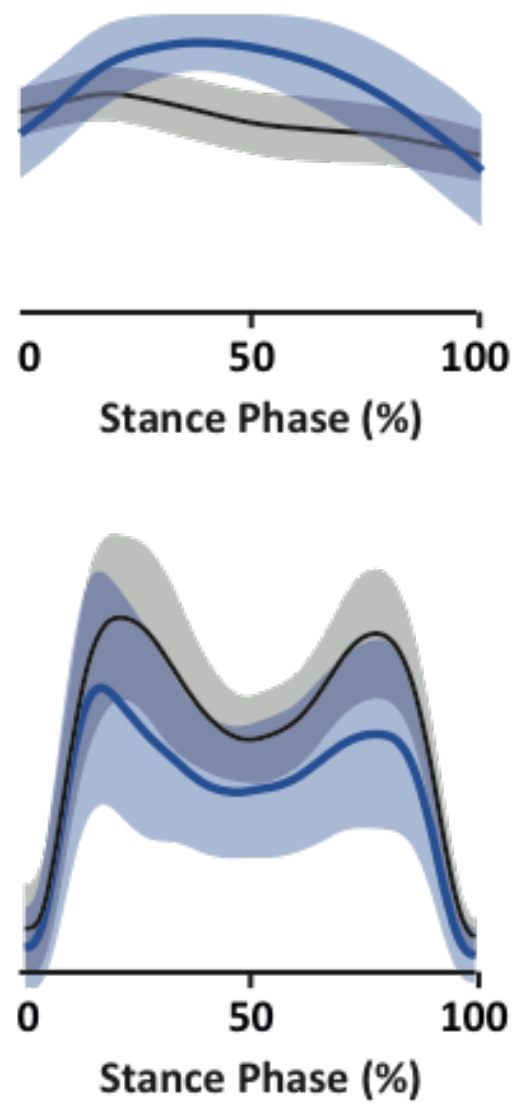
Table 1: Mean and standard deviation of variables for wide step width and narrow step width groups. Values represent mean \pm standard deviation. $K A M=$ Internal knee abduction moment, $\mathrm{COM}=$ centre of mass. $\mathrm{COG}=$ centre of gravity

\begin{tabular}{|c|c|c|c|c|c|}
\hline & & \multicolumn{2}{|c|}{ Wide step width } & \multicolumn{2}{|c|}{ Normal step width } \\
\hline & & $\begin{array}{c}\text { Normal trunk } \\
\text { lean walk }\end{array}$ & $\begin{array}{l}6^{\circ} \text { Trunk } \\
\text { lean }\end{array}$ & $\begin{array}{l}\text { Normal trunk } \\
\text { lean walk }\end{array}$ & $\begin{array}{l}6^{\circ} \text { Trunk } \\
\text { lean }\end{array}$ \\
\hline Gait Velocity (ms $\left.{ }^{-1}\right)$ & & $1.58 \pm 0.09$ & $1.60 \pm 0.09$ & $1.45 \pm 0.12$ & $1.47 \pm 0.08$ \\
\hline Step width (cm) & & $11.3 \pm 2.4$ & $24.7 \pm 5.5$ & $12.2 \pm 2.8$ & $13.7 \pm 4.7$ \\
\hline Peak trunk lean $\left({ }^{\circ}\right)$ & & $2.8 \pm 2.2$ & $7.7 \pm 2.7$ & $2.5 \pm 2.0$ & $7.8 \pm 2.4$ \\
\hline Time to peak trunk lean & stance) & $18.9 \pm 6.2$ & $43.5 \pm 14.0$ & $20.4 \pm 8.4$ & $26.9 \pm 12.2$ \\
\hline COM displacement (cm) & & $1.98 \pm 0.98$ & $3.53 \pm 1.05$ & $1.64 \pm 0.66$ & $2.62 \pm 1.74$ \\
\hline \multirow[t]{2}{*}{ KAM (Nm/kg) } & $1^{\text {st }}$ peak & $0.50 \pm 0.12$ & $0.41 \pm 0.15$ & $0.38 \pm 0.08$ & $0.30 \pm 0.10$ \\
\hline & $2^{\text {nd }}$ peak & $0.45 \pm 0.10$ & $0.32 \pm 0.13$ & $0.36 \pm 0.10$ & $0.35 \pm 0.15$ \\
\hline \multirow{2}{*}{$\begin{array}{l}\text { Absolute KAM impulse } \\
\text { stance phase ( } \mathrm{Nm} / \mathrm{kg} / \mathrm{s})\end{array}$} & $1^{\text {st }}$ half & $0.11 \pm 0.03$ & $0.08 \pm 0.04$ & $0.08 \pm 0.02$ & $0.06 \pm 0.02$ \\
\hline & $2^{\text {nd }}$ half & $0.09 \pm 0.02$ & $0.06 \pm 0.03$ & $0.07 \pm 0.02$ & $0.07 \pm 0.04$ \\
\hline
\end{tabular}


Figure 3: Frontal plane COM displacement for a full gait cycle (heel strike to heel strike). Each line represents the difference between normal walking and walking with the lateral trunk lean (LTL-NW). Graph displays means (solid lines) and standard deviations. The red line displays data for the narrow step width group and the blue represents the wide step width group. The COM displacement is greater in the WSW group despite both achieving the same degree of trunk lean.

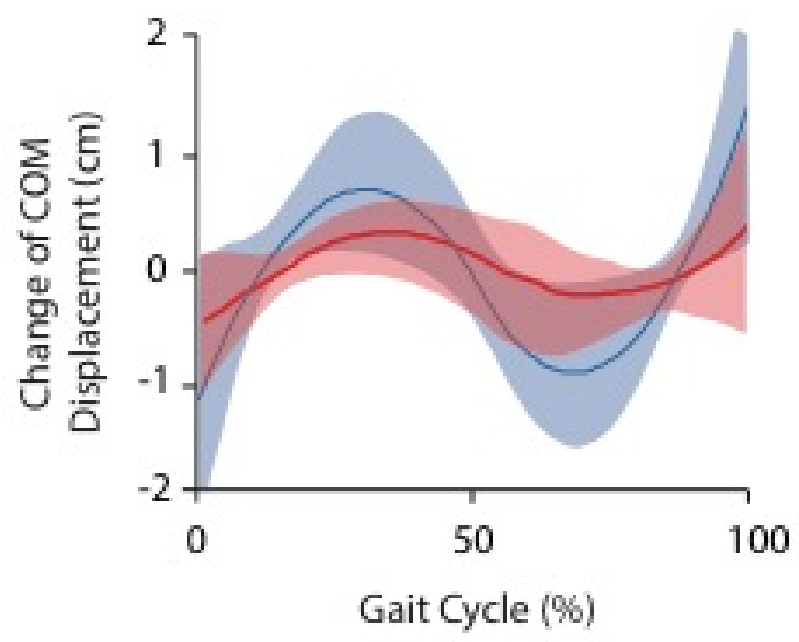

